\title{
ON STABLY FREE MODULES OVER AFFINE ALGEBRAS
}

\author{
JEAN FASEL, RAVI A. RAO, AND RICHARD G. SWAN
}

\begin{abstract}
If $X$ is a smooth affine variety of dimension $d$ over an algebraically closed field $k$, and if $(d-1) ! \in k^{\times}$then any stably trivial vector bundle of rank $(d-1)$ over $X$ is trivial. The hypothesis that $X$ is smooth can be weakened to $X$ is normal if $d \geq 4$.
\end{abstract}

\section{Contents}

1. Introduction

1.1. Conventions

1.2. Acknowledgements

2. Grothendieck-Witt groups

2.1. Preliminaries

2.2. Basic computations

3. The elementary symplectic Witt group

3.1. An exact sequence

4. Identification with $G W_{1}^{3}(R)$

4.1. The group $G W_{1}^{3}(R)$

4.2. The group $\mathrm{V}(R)$

4.3. The isomorphism

5. The Gersten-Grothendieck-Witt spectral sequence 10

6. Divisibility of $W_{E} \quad 11$

7. The main theorem 13

References $\quad 16$

\section{INTRODUCTION}

Let $R$ be an affine algebra of dimension $d$ over a field $k$. Recall that a projective $R$-module $P$ of rank $r$ is said to be cancellative if for any projective module $Q$ such that $P \oplus R^{n} \simeq Q \oplus R^{n}$ then $P \simeq Q$. A famous theorem by Bass and Schanuel asserts that any $P$ is cancellative if $r>d$ ([5, Theorem 9.3]). In general, this is the best possible bound as shown by the well known example of the tangent bundle of the real algebraic sphere of dimension 2 .

However, A.A. Suslin has shown in [33, 34] that if $k$ is algebraically closed, then any projective module $P$ of rank $d$ is cancellative. In [35, he further showed that if c.d. $(k) \leq 1$ then $R^{d}$ is cancellative provided $(d-1) ! \in k^{\times}$, thus proving that stably free modules of rank $\geq d$ are actually free in this setting. As a consequence of this

2000 Mathematics Subject Classification. Primary: 13C10; Secondary: 19G12, 19 G38.

Key words and phrases. stably free modules, affine algebras, Grothendieck-Witt groups, symplectic Witt groups. 
result, S.M. Bhatwadekar showed in $[8]$ that any projective module $P$ of rank $d$ is cancellative in this situation.

The natural question is to know under which conditions projective $R$-modules of rank $r<d$ are cancellative. A weaker question, asked by Suslin in [34], is to understand the conditions under which $R^{r}$ itself is cancellative, i.e. when stably free modules of rank $r$ are free.

In the same article, A.A. Suslin mentions that all stably free non free modules known to him are of rank $\leq(\operatorname{dim} R-1) / 2$. In response to this, in [19] N. Mohan Kumar gave a method of constructing a non-free stably free projective module of rank $d-1$ over the coordinate ring of certain basic open sets $D(f)$ of the affine space $\mathbb{A}_{k}^{d}$, for $d \geq 4$, where $k$ is of transcendence degree one over an algebraically closed field. Clearing "denominators" he could get a smooth, rational affine variety of dimension $d$ over an algebraically closed field and a rank $d-2$ stably free non-free projective module over it.

Thus the above question boils down to understand if stably free modules of rank $d-1$ over $R$ are free. In 14 the first named author made the initial breakthrough to Suslin's question by showing that over a smooth threefold $R$, any stably free rank two projective module is free if $6 \in R^{\times}$. In this article, we extend this result and give a final answer to Suslin's question (Theorem 7.5 in the text).

Theorem. Let $R$ be a d-dimensional normal affine algebra over an algebraically closed field $k$ such that $\operatorname{gcd}((d-1) !, \operatorname{char}(k))=1$. If $d=3$, suppose moreover that $R$ is smooth. Then every stably free $R$-module $P$ of rank $d-1$ is free.

The solution of this problem of Suslin is an important bridge between classical algebraic K-theory, higher Grothendieck-Witt groups, and Milnor K-theory. This paper highlights these connections which will play a critical role in future development of the subject.

We motivate our method by recollecting the salient points of Suslin's proof that stably free modules of rank $d$ are free over affine algebras of dimension $d$ over a field with c.d. $(k) \leq 1$ :

- In view of the cancellation theorem of Bass and Schanuel, any stably free module $P$ of rank $d$ corresponds to a unimodular row $\left(a_{1}, \ldots, a_{d+1}\right)$ of length $d+1$.

- By Swan's Bertini theorem [37 one can ensure, after an elementary transformation, that $C:=R /\left(a_{4}, \ldots, a_{d+1}\right)$ is a surface and $B:=R /\left(a_{3}, \ldots, a_{d+1}\right)$ is a smooth curve over $k$, provided $k$ is infinite (when $k$ is finite, the result follows from a result of Vaserstein).

- In this situation, there exists a well-defined map

$$
U m_{2}(B) / S L_{2}(B) \cap E S p(B) \rightarrow U m_{d+1}(R) / E_{d+1}(R)
$$

under which the class of $\left(\bar{a}_{1}, \bar{a}_{2}\right)$ is sent to the class of $\left(a_{1}, \ldots, a_{d+1}\right)$ (35. Corollary 2.3]).

- There is a natural bijection $U m_{2}(B) / S L_{2}(B) \cap E S p(B) \rightarrow K_{1} S p(B)$ and the forgetful map $K_{1} S p(B) \rightarrow S K_{1}(B)$ is an isomorphism ([35, Proposition 1.7]).

- The Brown-Gersten-Quillen spectral sequence yields an isomorphism $S K_{1}(B) \simeq$ $H^{1}\left(B, K_{2}\right)$, and the latter is a uniquely divisible group prime to the characteristic of $k$ ([35, Proposition 1.4, Theorem 1.8]).

- As a consequence of the above points, we can suppose up to elementary transformations that $\left(a_{1}, a_{2}, a_{3}, \ldots, a_{d+1}\right)=\left(b_{1}^{d !}, b_{2}, a_{3}, \ldots, a_{d+1}\right)$. The right hand term is the first row of an invertible matrix ([34, Theorem 2]) and therefore $P$ is free. 
In [35, Remark 2.5], Suslin observes that this method might generalize to a proof that stably free modules of rank $d-1$ are free. Indeed, our method follows closely his ideas. More precisely, we proceed as follows:

- In view of Suslin's cancellation theorem, any stably free module $P$ of rank $d-1$ corresponds to a unimodular row $\left(a_{1}, \ldots, a_{d}\right)$ of length $d$.

- Under our hypothesis, one can ensure using Swan's Bertini theorem that $B:=$ $R /\left(a_{4}, \ldots, a_{d}\right)$ is a smooth threefold over $k$ (performing some elementary transformations if necessary).

- There is a natural map

$$
U m_{3}(B) / E_{3}(B) \rightarrow U m_{d}(R) / E_{d}(R)
$$

such that $\left(\bar{a}_{1}, \bar{a}_{2}, \bar{a}_{3}\right) \mapsto\left(a_{1}, a_{2}, a_{3}, \ldots, a_{d}\right)$.

- Using Rao-van der Kallen's result ([27, Corollary 3.5]), the Vaserstein symbol

$$
V: U m_{3}(B) / E_{3}(B) \rightarrow W_{E}(B)
$$

is a bijection, where $W_{E}(B)$ is the elementary symplectic Witt group as defined in [40]. Thus $U m_{3}(B) / E_{3}(B)$ is endowed with the structure of an abelian group.

- There is an isomorphism $W_{E}(B) \simeq G W_{1, \text { red }}^{3}(B)$, where $G W_{1, \text { red }}^{3}(B)$ is a reduced Grothendieck-Witt group of $B$.

- Using the Gersten-Grothendieck-Witt spectral sequence, the group $G W_{1, \text { red }}^{3}(B)$ is proved to be isomorphic to $H^{2}\left(B, K_{3}\right)$, and the latter is shown to be divisible using the Merkurjev-Suslin theorem on $K_{2}^{M}([20])$.

- As $\sqrt{-1} \in k$, we can use Rao's antipodal Lemma [25. Lemma 1.3.1] and the above points to suppose that $\left(a_{1}, a_{2}, a_{3}, \ldots, a_{d}\right)=\left(b_{1}^{(d-1) !}, b_{2}, a_{3}, \ldots, a_{d}\right)$ up to elementary transformations. This allows to conclude as in Suslin's proof.

The organization of the paper is as follows. Section 2 gives the basic definitions and results of the theory of Grothendieck-Witt groups as developed by M. Schlichting. In Section 3, we recall the definition of the elementary symplectic Witt group $W_{E}$. We also recall an exact sequence, later used in Section 4 to show that the elementary symplectic Witt group is precisely a Grothendieck-Witt group (Theorem 4.4 in the text). This is one of the main observations of this work, as it allows to use the powerful techniques of the theory of Grothendieck-Witt groups to study $W_{E}$. Among these techniques, one of the most useful is the Gersten-Grothendieck-Witt spectral sequence. It is the analogue of the classical Brown-Gersten-Quillen spectral sequence in $K$-theory. For a smooth affine threefold $R$ over an algebraically closed field, this sequence is easy to analyse and allows to prove that $W_{E}(R) \simeq H^{2}\left(R, K_{3}\right)$. In Section [6, we show that this cohomology group is divisible. We claim no originality in this computation, which seems to be well-known to specialists. All the pieces fall together in Section 7 where we finally prove our main theorem, following the path described above. As a consequence, we derive from the main result a few prestabilization results for the functor $K_{1}$.

It must be observed that the question whether every projective module of rank $d-1$ over an algebra of dimension $d$ over an algebraically closed field $k$ is cancellative is still open. As opposed to the case of projective modules of rank $d$, the full result is not an immediate consequence of the fact that $R^{d-1}$ is cancellative. We hope to treat this question somewhere in further work.

1.1. Conventions. Every ring is commutative with 1 , any algebra is of finite type over some field. For any abelian group $G$ and any $n \in \mathbb{N}$, we denote by $\{n\} G$ the 
subgroup of $n$-torsion elements in $G$. All the fields considered are of characteristic different from 2. If $X$ is a scheme and $x_{p} \in X^{(p)}$, we denote by $\mathfrak{m}_{p}$ the maximal ideal in $\mathcal{O}_{X, x_{p}}$ and by $k\left(x_{p}\right)$ its residue field. Finally $\omega_{x_{p}}$ will denote the dual of the $k\left(x_{p}\right)$-vector space $\wedge^{p}\left(\mathfrak{m}_{p} / \mathfrak{m}_{p}^{2}\right)$ (which is one-dimensional if $X$ is regular at $\left.x_{p}\right)$. If $M$ and $N$ are square matrices, we denote by $M \perp N$ the matrix $\left(\begin{array}{cc}M & 0 \\ 0 & N\end{array}\right)$.

1.2. Acknowledgements. The first author wishes to thank the Swiss National Science Foundation, grant PP00P2_129089/1, for support.

\section{Grothendieck-Witt Groups}

2.1. Preliminaries. In this section, we recall a few basic facts about GrothendieckWitt groups. These are a modern version of Hermitian $K$-theory. The general reference here is the work of M. Schlichting ([29], 30]). Since we use GrothendieckWitt groups only for affine schemes, we restrict to this case.

Let $R$ be a ring with $2 \in R^{\times}$. Let $\mathcal{P}(R)$ be the category of finitely generated projective $R$-modules and $C h^{b}(R)$ be the category of bounded complexes of objects in $\mathcal{P}(R)$. It carries the structure of an exact category, by saying that an exact sequence of complexes is exact if it is exact in $\mathcal{P}(R)$ degreewise. For any line bundle $L$ on $R$, the duality $\operatorname{Hom}_{R}(,, L)$ on $\mathcal{P}(R)$ induces a duality $\sharp_{L}$ on $C h^{b}(R)$ and the canonical identification of a projective module with its double dual gives a natural isomorphism of functors $\varpi_{L}: 1 \rightarrow \sharp_{L} \sharp_{L}$. One can also define a weakequivalence in $C h^{b}(R)$ to be a quasi-isomorphism of complexes. This shows that $\left(C h^{b}(R), q i s, \sharp_{L}, \varpi_{L}\right)$ is an exact category with weak-equivalences and duality in the sense of [30, §2.3] (see also [loc. cit., §6.1]). The translation functor (to the left) $T: C h^{b}(R) \rightarrow C h^{b}(R)$ yields new dualities $\sharp_{L}^{n}:=T^{n} \circ \sharp_{L}$ and canonical isomorphisms $\varpi_{L}^{n}:=(-1)^{n(n+1) / 2} \varpi_{L}$.

To any exact category with weak-equivalences and duality, Schlichting associates a space $\mathcal{G W}$ and defines the (higher) Grothendieck-Witt groups to be the homotopy groups of that space $([30, \S 2.11])$. More precisely:

Definition 2.1. We denote by $G W_{i}^{j}(R, L)$ the group $\pi_{i} \mathcal{G W}\left(C h^{b}(R), q i s, \sharp_{L}^{j}, \varpi_{L}^{j}\right)$. If $L=R$, we simply put $G W_{i}^{j}(R)=G W_{i}^{j}(R, R)$.

Of course, the Grothendieck-Witt groups coincide with Hermitian $K$-theory ([17, [18]), at least when $2 \in R^{\times}([29$, remark 4.16], see also [16]). In particular, $G W_{i}^{0}(R)=K_{i} O(R)$ and $G W_{i}^{2}(R)=K_{i} S p(R)$. The twisted groups coincide with Karoubi $U$ groups, i.e. $G W_{i}^{1}(R)={ }_{-1} U_{i}(R)$ and $G W_{i}^{3}(R)=U_{i}(R)$.

The basic tool in the study of Grothendieck-Witt groups is the fundamental exact sequence ([18, [16, Definition 3.6]; see also [13, Theorem 8]):

$$
\ldots \rightarrow G W_{i}^{j}(R) \stackrel{f}{\longrightarrow} K_{i}(R) \stackrel{H}{\longrightarrow} G W_{i}^{j+1}(R) \stackrel{\delta}{\longrightarrow} G W_{i-1}^{j}(R) \stackrel{f}{\longrightarrow} K_{i-1}(R) \rightarrow \ldots
$$

The fundamental exact sequence ends with Witt groups as defined by Balmer in [2], see [29, Lemma 4.13] or [13, Theorem 9]:

$$
\ldots \longrightarrow G W^{i}(R) \stackrel{f}{\longrightarrow} K_{0}(R) \stackrel{H}{\longrightarrow} G W^{i+1}(R) \longrightarrow W^{i+1}(R) \longrightarrow 0 .
$$

The homomorphism $f$ is called the forgetful homomorphism and the homomorphism $H$ is called the hyperbolic homomorphism. The object of the next section is to give 
some basic computations of Grothendieck-Witt groups of fields that will be used later.

\subsection{Basic computations.}

Lemma 2.2. For any field $F$ of characteristic different from 2, the groups $G W_{i}^{i+1}(F)$ are trivial for $i=0,1,2$.

Proof. First observe that by definition $G W_{2}^{2}(F)=K_{2} S p(F)$. Recall from 36, Corollary 6.4] that there is a homomorphism $K_{2} S p(F) \rightarrow I^{2}(F)$ such that the following diagram is a fibre product

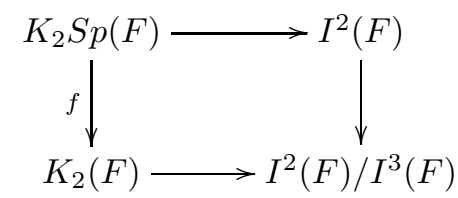

where the bottom horizontal map is the homomorphism defined by Milnor in 22 , Theorem 4.1] and the right vertical map is the quotient map. It follows that the forgetful map $G W_{2}^{2}(F) \rightarrow K_{2}(F)$ is surjective and the exact sequence

$$
G W_{2}^{2}(F) \stackrel{f}{\longrightarrow} K_{2}(F) \stackrel{H}{\longrightarrow} G W_{2}^{3}(F) \longrightarrow G W_{1}^{2}(F)
$$

shows that it suffices to prove that $G W_{1}^{2}(F)=0$ to have $G W_{2}^{3}(F)=0$. This is clear since $G W_{1}^{2}(F)=K_{1} S p(F)=0$. To conclude, we are left to prove that $G W_{0}^{1}(F)=0$. But this is clear since the sequence

$$
G W(F) \stackrel{f}{\longrightarrow} K_{0}(F) \stackrel{H}{\longrightarrow} G W_{0}^{1}(F) \longrightarrow W^{1}(F)
$$

is exact and $W^{1}(F)=0$ ([3, Proposition 5.2]).

Lemma 2.3. The hyperbolic functor $K_{i}(F) \rightarrow G W_{i}^{i+2}(F)$ is an isomorphism for $i=0,1$.

Proof. This is obvious and well known for $G W^{2}(F)=K_{0} S p(F)$. For $G W_{1}^{3}(F)$, it suffices to use the fundamental exact sequence and Lemma 2.2

Lemma 2.4. The forgetful functor induces surjections $f: G W_{i}^{i-1}(F) \rightarrow\{2\} K_{i}(F)$ for $i=1,2$. Moreover, if $F$ is algebraically closed then $f: G W_{1}^{0}(F) \rightarrow\{ \pm 1\}$ is an isomorphism.

Proof. For any field $F$, the fundamental exact sequence

$$
G W_{i}^{i-1}(F) \stackrel{f}{\longrightarrow} K_{i}(F) \stackrel{H}{\longrightarrow} G W_{i}^{i}(F)
$$

and the proof of Lemma 2.2 yield surjective homomorphisms

$$
G W_{i}^{i-1}(F) \rightarrow\{2\} K_{i}(F)
$$

for $i=1,2$. Suppose now that $F$ is algebraically closed. The fundamental exact sequence

$$
G W_{1}^{3}(F) \stackrel{f}{\rightarrow} K_{1}(F) \stackrel{H}{\rightarrow} G W_{1}^{0}(F) \rightarrow G W^{3}(F) \stackrel{f}{\rightarrow} K_{0}(F) \stackrel{H}{\rightarrow} G W(F)
$$

and Lemma 2.3 give an exact sequence

$$
0 \longrightarrow K_{1}(F) / 2 \longrightarrow G W_{1}^{0}(F) \longrightarrow G W^{3}(F) \longrightarrow 0 \text {. }
$$


Since $F$ is algebraically closed, the left term is trivial. Now $G W^{3}(F)=\mathbb{Z} / 2$ by $[12$, Lemma 4.1].

\section{The ElEmentary SyMPlectic Witt GRoup}

Let $R$ be a ring (with $2 \in R^{\times}$). We first briefly recall the definition of the elementary symplectic Witt group considered in [40]. For any $n \in \mathbb{N}$, let $S_{2 n}^{\prime}(R)$ be the set of skew-symmetric matrices in $G L_{2 n}(R)$. For any $r \in \mathbb{N}$, Let $\psi_{2 r}$ be the matrix defined inductively by

$$
\psi_{2}:=\left(\begin{array}{cc}
0 & 1 \\
-1 & 0
\end{array}\right)
$$

and $\psi_{2 r}:=\psi_{2} \perp \psi_{2 r-2}$. Observe that $\psi_{2 r} \subset S_{2 r}^{\prime}$. For any $m<n$, there is an obvious inclusion of $S_{2 m}^{\prime}(R)$ in $S_{2 n}^{\prime}(R)$ defined by $G \mapsto G \perp \psi_{2 n-2 m}$. We define $S^{\prime}(R)$ as the set $\cup S_{2 n}^{\prime}(R)$. There is an equivalence relation on $S^{\prime}(R)$ defined as follows:

If $G \in S_{2 n}^{\prime}(R)$ and $G^{\prime} \in S_{2 m}^{\prime}(R)$, then $G \sim G^{\prime}$ if and only if there exists $t \in \mathbb{N}$ and $E \in E_{2(m+n+t)}(R)$ such that

$$
G \perp \psi_{2(m+t)}=E^{t}\left(G^{\prime} \perp \psi_{2(n+t)}\right) E .
$$

Observe that $S^{\prime}(R) / \sim$ has the structure of an abelian group, with $\perp$ as operation and $\psi_{2}$ as neutral element. One can also consider the subsets $S_{2 n}(R) \subset S_{2 n}^{\prime}(R)$ of invertible skew-symmetric matrices with Pfaffian equal to 1 . If $S(R):=\cup S_{2 n}(R)$, it is easy to see that $\sim$ induces an equivalence relation on $S(R)$ and that $S(R) / \sim$ is also a group.

Definition 3.1. We denote by $W_{E}^{\prime}(R)$ the group $S^{\prime}(R) / \sim$ and by $W_{E}(R)$ the group $S(R) / \sim$. The latter is called elementary symplectic Witt group.

3.1. An exact sequence. In this section, we prove a few basic facts about the elementary symplectic Witt group and obtain a useful exact sequence (see [14, §2]). If $G$ is a skew-symmetric invertible matrix of size $2 n$, it can be seen as a skewsymmetric form on $R^{2 n}$. This yields a map $\varphi_{2 n}: S_{2 n}^{\prime}(R) \rightarrow \widetilde{K}_{0} S p(R)$, where the latter is the reduced symplectic $K_{0}$ of the ring $R$. This map passes to the limit and preserves the equivalence relation $\sim$ (as well as the orthogonal sum $\perp$ ). Hence we get a homomorphism

$$
\varphi: W_{E}^{\prime}(R) \rightarrow \widetilde{K}_{0} S p(R)
$$

We define a map

$$
\eta_{2 n}: G L_{2 n}(R) \rightarrow S^{\prime}(R)
$$

by $\eta_{2 n}(G)=G^{t} \psi_{2 n} G$ for any $G \in G L_{2 n}(R)$, and a map

$$
\eta_{2 n+1}: G L_{2 n+1}(R) \rightarrow S^{\prime}(R)
$$

by $\eta_{2 n+1}(G)=(G \perp 1)^{t} \psi_{2(n+1)}(G \perp 1)$ for any $G \in G L_{2 n+1}(R)$. These maps obviously pass to the limit, and we obtain a map $\eta: G L(R) \rightarrow W_{E}^{\prime}(R)$ after composing with the projection $S^{\prime}(R) \rightarrow W_{E}^{\prime}(R)$. Using Whitehead Lemma ([23, proof of Lemma 2.5]), it is not hard to see that $\eta$ induces a homomorphism

$$
\eta: K_{1}(R) \rightarrow W_{E}^{\prime}(R) .
$$


Proposition 3.2. The sequence

$$
K_{1} S p(R) \stackrel{f^{\prime}}{\longrightarrow} K_{1}(R) \stackrel{\eta}{\longrightarrow} W_{E}^{\prime}(R) \stackrel{\varphi}{\longrightarrow} \widetilde{K}_{0} S p(R) \stackrel{f}{\longrightarrow} \widetilde{K}_{0}(R)
$$

is exact, where $f^{\prime}$ is the homomorphism induced by $S p_{2 n} \subset S L_{2 n}$ for any $n, f$ is the forgetful homomorphism and $\widetilde{K}_{0}(R)$ is the reduced $K_{0}$ of $R$.

Proof. First, a straightforward computation shows that this sequence is a complex. We now check that it is exact.

It is well known that any element of $\widetilde{K}_{0} S p(R)$ is of the form $[P, \phi]$ for some projective module $P$ and some skew-symmetric form $\phi: P \rightarrow P^{\vee}$ ([7, Proposition $2])$. But $f(P, \phi)=0$ if and only if $P$ is stably free and adding a suitable $H\left(R^{s}\right)$ we see that $P$ is given by a skew-symmetric matrix on some $R^{2 n}$.

Let $M$ be a skew-symmetric (invertible) matrix such that $\varphi(M)=0$. Adding if necessary some multiple of $\psi_{2}$, we have $M=G^{t} \psi_{2 n} G$ for some $n \in \mathbb{N}$ and therefore $M=\eta(G)$.

If $G \in K_{1}(R)$ is such that $\eta(G)=0$, we can suppose that $G \in G L_{2 n}(R)$ for some $n \in \mathbb{N}$ and the triviality of $\eta(G)$ is expressed as $G^{t} \psi_{2 n} G \sim \psi_{2}$. This translates as

$$
\psi_{2(n+s)}=E^{t}\left(\left(G^{t} \psi_{2 n} G\right) \perp \psi_{2 s}\right) E=E^{t}\left(\left(G \perp I_{2 s}\right)^{t} \psi_{2(n+s)}\left(G \perp I_{2 s}\right)\right) E
$$

for some $s \in \mathbb{N}$ and some $E \in E_{2(n+s)}(R)$. Therefore $\left(G \perp I_{2 s}\right) E \in S p_{2(n+s)}(R)$.

\section{Identification with $G W_{1}^{3}(R)$}

4.1. The group $G W_{1}^{3}(R)$. Recall from Section 2.1 that $G W_{1}^{3}(R)$ is defined to be the group $\pi_{1} \mathcal{G W}\left(C h^{b}(R), q i s, \sharp^{3}, \varpi^{3}\right)$.

Consider the category $\mathcal{S F}(R)$ of stably-free $R$-modules of finite rank and its subcategory $\mathcal{F}(R)$ of free $R$-modules of finite rank. Let $C h_{\mathrm{sf}}^{b}(R)\left(\right.$ resp. $\left.C h_{\text {free }}^{b}(R)\right)$ be the category $C h_{\mathrm{sf}}^{b}(R)$ of bounded complexes of objects of $\mathcal{S F}(R)($ resp. $\mathcal{F}(R))$. Restricting the duality, the canonical isomorphism and the quasi-isomorphisms to $C h_{\mathrm{sf}}^{b}(R)$ and $C h_{\text {free }}^{b}(R)$, we see that both categories are also exact categories with weak-equivalences and duality. If we denote by $G W_{1, \mathrm{sf}}^{3}(R)$ the Grothendieck-Witt group obtained from the category $C h_{\mathrm{sf}}^{b}(R)$ and $G W_{1, \text { free }}^{3}(R)$ the Grothendieck-Witt group obtained from the category $C h_{\text {free }}^{b}(R)$, then the natural map $C h_{\mathrm{sf}}^{b}(R) \rightarrow$ $C h^{b}(R)$ induces an isomorphism

$$
G W_{1, \mathrm{sf}}^{3}(R) \rightarrow G W_{1}^{3}(R)
$$

by [30, Theorem 7], while the natural map $C h_{\text {free }}^{b}(R) \rightarrow C h_{\mathrm{sf}}^{b}(R)$ induces an isomorphism

$$
G W_{1, \text { free }}^{3}(R) \rightarrow G W_{1, \mathrm{sf}}^{3}(R)
$$

by [30, Lemma 9].

Recall moreover that the group $G W_{1}^{3}(R)$ fits in the fundamental exact sequence

$$
K_{1} S p(R) \stackrel{f^{\prime}}{\longrightarrow} K_{1}(R) \stackrel{H}{\longrightarrow} G W_{1}^{3}(R) \stackrel{\delta}{\longrightarrow} K_{0} S p(R) \stackrel{f}{\longrightarrow} K_{0}(R)
$$

which gives an exact sequence

$$
K_{1} S p(R) \stackrel{f^{\prime}}{\longrightarrow} K_{1}(R) \stackrel{H}{\longrightarrow} G W_{1}^{3}(R) \stackrel{\delta}{\longrightarrow} \widetilde{K}_{0} S p(R) \stackrel{f}{\longrightarrow} \widetilde{K}_{0}(R)
$$


4.2. The group $\mathbf{V}(R)$. Consider the set of triples $\left(L, f_{0}, f_{1}\right)$, where $L$ is a free module and $f_{i}: L \simeq L^{\vee}$ are skew-symmetric isomorphisms $(i=0,1)$. Two triples $\left(L, f_{0}, f_{1}\right)$ and $\left(L^{\prime}, f_{0}^{\prime}, f_{1}^{\prime}\right)$ are isometric if there exists an isomorphism $\alpha: L \rightarrow L^{\prime}$ such that $f_{i}=\alpha^{\vee} f_{i}^{\prime} \alpha$ for $i=0,1$. We denote by $\left[L, f_{0}, f_{1}\right]$ the isometry class of a triple $\left(L, f_{0}, f_{1}\right)$. The orthogonal sum

$$
\left[L, f_{0}, f_{1}\right] \perp\left[L^{\prime}, f_{0}^{\prime}, f_{1}^{\prime}\right]:=\left[L \oplus L^{\prime}, f_{0} \perp f_{0}^{\prime}, f_{1} \perp f_{1}^{\prime}\right]
$$

endows the set $\mathrm{MV}^{\prime}(R)$ of isometry classes of triples $\left[L, f_{0}, f_{1}\right]$ with a structure of a monoid and we can consider its Grothendieck group $V^{\prime}(R)$. We denote by $V(R)$ the quotient of $V^{\prime}(R)$ by the subgroup generated by the relations

$$
\left[L, f_{0}, f_{1}\right]+\left[L, f_{1}, f_{2}\right]-\left[L, f_{0}, f_{2}\right] .
$$

The group $V(R)$ is naturally isomorphic to $G W_{1, \text { free }}^{3}(R)$ by [29], and the latter is isomorphic to $G W_{1}^{3}(R)$ as seen above.

4.3. The isomorphism. Following [40, §3], we define for any $n \in \mathbb{N}$ a matrix $\sigma_{2 n}: R^{2 n} \rightarrow R^{2 n}$ inductively by

$$
\sigma_{2}:=\left(\begin{array}{cc}
0 & 1 \\
1 & 0
\end{array}\right)
$$

and $\sigma_{2 n}:=\sigma_{2} \perp \sigma_{2 n-2}$. Observe that $\sigma_{2 n}^{2}=I d_{2 n}$.

Let $\left(L, f_{0}, f_{1}\right)$ be a triple as in the above section, with $L$ free. Choose an isomorphism $g: R^{2 n} \rightarrow L$ and consider the isomorphism

$$
\left(g^{\vee} f_{1} g\right) \perp \sigma_{2 n}\left(g^{\vee} f_{0} g\right)^{-1} \sigma_{2 n}^{\vee}: R^{2 n} \oplus\left(R^{2 n}\right)^{\vee} \rightarrow\left(R^{2 n}\right)^{\vee} \oplus R^{2 n} .
$$

It is clearly skew-symmetric and we can consider its class in $W_{E}^{\prime}(R)$.

Lemma 4.1. The class of $\left(g^{\vee} f_{1} g\right) \perp \sigma_{2 n}\left(g^{\vee} f_{0} g\right)^{-1} \sigma_{2 n}^{\vee}$ in $W_{E}^{\prime}(R)$ does not depend on $g$.

Proof. If $h: R^{2 n} \rightarrow L$ is another isomorphism, let $a:=h^{-1} g \perp \sigma_{2 n}^{\vee}\left(g^{-1} h\right)^{\vee} \sigma_{2 n}^{\vee}$. Then we have

$$
\left(g^{\vee} f_{1} g\right) \perp\left(-g^{\vee} f_{0} g\right)^{-1}=a^{\vee}\left[\left(h^{\vee} f_{1} h\right) \perp \sigma_{2 n}\left(h^{\vee} f_{0} h\right)^{-1} \sigma_{2 n}^{\vee}\right] a .
$$

It is then sufficient to prove that the conjugation by $m \perp \sigma_{2 n}^{\vee}\left(m^{-1}\right)^{\vee} \sigma_{2 n}^{\vee}$ is trivial in $W_{E}^{\prime}(R)$ for any automorphism $m$ of $R^{2 n}$. To achieve this, we adapt the arguments of [4, Lemma 4.1.3].

Let $h_{4 n}: R^{2 n} \oplus\left(R^{2 n}\right)^{\vee} \rightarrow\left(R^{2 n}\right)^{\vee} \oplus R^{2 n}$ be given by the matrix $\left(\begin{array}{cc}0 & \sigma_{2 n}^{\vee} \\ -\sigma_{2 n} & 0\end{array}\right)$. We have

$$
\left(\begin{array}{cc}
\left(m^{-1}\right)^{\vee} & 0 \\
0 & \sigma_{2 n} m \sigma_{2 n}
\end{array}\right) \cdot h_{4 n} \cdot\left(\begin{array}{cc}
m^{-1} & 0 \\
0 & \sigma_{2 n}^{\vee} m^{\vee} \sigma_{2 n}^{\vee}
\end{array}\right)=h_{4 n} .
$$

Adding $h_{4 n}$ to $\left(h^{\vee} f_{1} h\right) \perp \sigma_{2 n}\left(h^{\vee} f_{0} h\right)^{-1} \sigma_{2 n}^{\vee}$, we see therefore that we have to prove that $m \perp \sigma_{2 n}^{\vee}\left(m^{-1}\right)^{\vee} \sigma_{2 n}^{\vee} \perp m^{-1} \perp \sigma_{2 n}^{\vee} m^{\vee} \sigma_{2 n}^{\vee}$ is an elementary matrix. This is clear by Whitehead Lemma ([23, proof of Lemma 2.5]).

We set $\zeta\left(L, f_{0}, f_{1}\right)$ to be the class of $\left(g^{\vee} f_{1} g\right) \perp \sigma_{2 n}\left(g^{\vee} f_{0} g\right)^{-1} \sigma_{2 n}^{\vee}$ in $W_{E}^{\prime}(R)$ for any isomorphism $g: R^{2 n} \rightarrow L$. Next we deal with isometries:

Lemma 4.2. If $\left(L, f_{0}, f_{1}\right)$ and $\left(L^{\prime}, f_{0}^{\prime}, f_{1}^{\prime}\right)$ are isometric, then we have an equality $\zeta\left(L, f_{0}, f_{1}\right)=\zeta\left(L^{\prime}, f_{0}^{\prime}, f_{1}^{\prime}\right)$ in $W_{E}^{\prime}(R)$. 
Proof. If $g: R^{2 n} \rightarrow L$ is an isomorphism, and $\alpha: L \rightarrow L^{\prime}$ is an isometry then $\alpha g: R^{2 n} \rightarrow L^{\prime}$ is an isomorphism and the result follows from $\alpha^{\vee} f_{i}^{\prime} \alpha=f_{i}$ for $i=0,1$.

Hence we see that the class $\zeta\left(\left[L, f_{0}, f_{1}\right]\right)$ is well defined. It is easy to check that it respects the direct sum, and thus we obtain a homomorphism

$$
\zeta: V^{\prime}(R) \rightarrow W_{E}^{\prime}(R)
$$

Proposition 4.3. The homomorphism $\zeta: V^{\prime}(R) \rightarrow W_{E}^{\prime}(R)$ induces a homomorphism

$$
\zeta: V(R) \rightarrow W_{E}^{\prime}(R)
$$

Proof. By definition of $\zeta$, it suffices to check that

$$
\zeta\left(\left[A^{2 n}, f_{0}, f_{1}\right]\right)+\zeta\left(\left[A^{2 n}, f_{1}, f_{2}\right]\right)=\zeta\left(\left[A^{2 n}, f_{0}, f_{2}\right]\right)
$$

in $W_{E}^{\prime}(R)$. The left-hand term is equal to

$$
f_{1} \perp \sigma_{2 n} f_{0}^{-1} \sigma_{2 n}^{\vee} \perp f_{2} \perp \sigma_{2 n} f_{1}^{-1} \sigma_{2 n}^{\vee}
$$

and it suffices therefore to prove that $f_{1} \perp \sigma_{2 n} f_{1}^{-1} \sigma_{2 n}^{\vee}$ is elementary equivalent to some $\psi_{2 n}$ to conclude. Since $\sigma_{2 n} f_{1}^{-1} \sigma_{2 n}^{\vee}$ is precisely the inverse of $f_{1}$ in $W_{E}(R)$ by [40, §3], we are done.

It remains to prove that $\zeta$ is an isomorphism, but all the work is almost done.

Theorem 4.4. Let $R$ be a ring with $2 \in R^{\times}$. The following diagram commutes

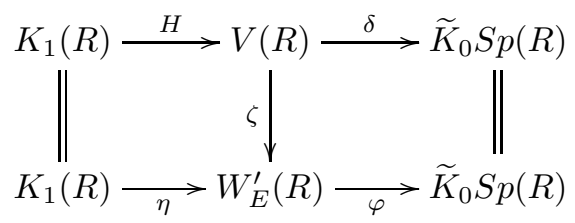

and $\zeta$ is an isomorphism.

Proof. We first prove that the diagram commutes. Let $a \in K_{1}(R)$ be represented by a matrix $G \in \mathrm{GL}_{2 n}(R)$. Then $H$ is given by $H(a)=\left[A^{2 n}, \psi_{2 n}, G^{\vee} \psi_{2 n} G\right.$ ] (see for instance [17]). Since $\sigma_{2 n}\left(\psi_{2 n}\right)^{-1} \sigma_{2 n}^{\vee}=\psi_{2 n}$, the left hand square commutes. If $\left[L, f_{0}, f_{1}\right]$ is in $V(R)$, then $\delta\left(\left[L, f_{0}, f_{1}\right]\right)=\left[L, f_{1}\right]-\left[L, f_{0}\right]$ in $\widetilde{K}_{0} S p(R)$. On the other hand,

$$
-\left[L, f_{0}\right]=\left[R^{2 n},-g^{\vee} f_{0} g\right]=\left[\left(R^{2 n}\right)^{\vee},\left(g^{\vee} f_{0} g\right)^{-1}\right]=\left[\left(R^{2 n}\right)^{\vee}, \sigma_{2 n}\left(g^{\vee} f_{0} g\right)^{-1} \sigma_{2 n}^{\vee}\right]
$$

and the right-hand square also commutes.

It follows then from Proposition 3.2 and the five lemma that $\zeta$ is an isomorphism.

This theorem shows that $W_{E}^{\prime}(R)$ inherits all the good properties of $G W_{1}^{3}(R)$. 


\section{The Gersten-Grothendieck-Witt spectral sequence}

Let $X$ be a regular scheme. Recall from [13, Theorem 25] that the GerstenGrothendieck-Witt spectral sequence is a spectral sequence $E(n)$ defined for any $n \in \mathbb{Z}$ converging to $G W_{n-*}^{n}(X)$ with terms on page 1 of the form (recall our conventions about $\left.\omega_{x_{p}}\right)$ :

$$
E(n)_{1}^{p, q}=\bigoplus_{x_{p} \in X^{(p)}} G W_{n-p-q}^{n-p}\left(k\left(x_{p}\right), \omega_{x_{p}}\right) .
$$

By construction of the corresponding spectral sequences, the forgetful homomorphism and the hyperbolic homomorphism induce morphisms of spectral sequences between the Gersten-Grothendieck-Witt spectral sequence and the Brown-GerstenQuillen spectral sequence in $K$-theory.

Proposition 5.1. Let $X$ be a smooth affine threefold over an algebraically closed field $k$. Then the Gersten-Grothendieck-Witt spectral sequence $E(3)^{p, q}$ yields an isomorphism

$$
W_{E}(X) \simeq H^{2}\left(X, K_{3}\right) .
$$

Proof. First remark that the line $q=1$ is trivial by Lemma 2.2 The line $q=2$ is as follows:

$$
G W_{1}^{3}(k(X), \omega) \longrightarrow \bigoplus_{x_{1} \in X^{(1)}} G W^{2}\left(k\left(x_{1}\right), \omega_{x_{1}}\right) \longrightarrow 0
$$

Lemma 2.3 shows that this is isomorphic, via $H$, to

$$
K_{1}(k(X)) \longrightarrow \bigoplus_{x_{1} \in X^{(1)}} K_{0}\left(k\left(x_{1}\right)\right) \longrightarrow 0
$$

whose homology at degree 0 is just $\mathcal{O}_{X}(X)^{\times}$. Now the Pfaffian homomorphism $G W_{1}^{3}(X) \rightarrow \mathcal{O}_{X}(X)^{\times}$is clearly split, and we see that the kernel of the edge homomorphism $G W_{1}^{3}(X) \rightarrow E(3)_{\infty}^{0,2}$ is precisely $W_{E}(X)$.

We now show that $E(3)_{\infty}^{2,0} \simeq H^{2}\left(X, K_{3}\right)$. But $E(3)_{\infty}^{2,0}$ is the homology of the complex

$$
\bigoplus_{x_{1} \in X^{(1)}} G W_{2}^{2}\left(k\left(x_{1}\right), \omega_{x_{1}}\right) \rightarrow \bigoplus_{x_{2} \in X^{(2)}} G W_{1}^{1}\left(k\left(x_{2}\right), \omega_{x_{2}}\right) \rightarrow \bigoplus_{x_{3} \in X^{(3)}} G W\left(k\left(x_{1}\right), \omega_{x_{1}}\right) .
$$

We use the forgetful functor to compare this sequence with the corresponding sequence

$$
\bigoplus_{x_{1} \in X^{(1)}} K_{2}\left(k\left(x_{1}\right)\right) \rightarrow \bigoplus_{x_{2} \in X^{(2)}} K_{1}\left(k\left(x_{2}\right)\right) \rightarrow \bigoplus_{x_{3} \in X^{(3)}} K_{0}\left(k\left(x_{1}\right)\right)
$$

in $K$-theory. After dévissage, the forgetful functor and the proof of Lemma 2.2 yield exact sequences

$$
0 \longrightarrow I^{4-p}(F) \longrightarrow G W_{3-p}^{3-p}(F) \stackrel{f}{\longrightarrow} K_{3-p}(F) \longrightarrow 0
$$

for any field $F$ and for $p=1,2,3$. Now if $x_{p} \in X^{(p)}$, then $\operatorname{cd}\left(k\left(x_{p}\right)\right) \leq 3-p$ by [31, $\S 4.2$, Proposition 11]. Hence $H^{4-p}\left(k\left(x_{p}\right), \mu_{2}\right)=0$ and the latter is isomorphic to $I^{4-p}\left(k\left(x_{p}\right)\right) / I^{5-p}\left(k\left(x_{p}\right)\right)$ by [24, Theorem 4.1] and [41, Theorem 7.4]. 
The Arason-Pfister Hauptsatz [1] then shows that $I^{4-p}\left(k\left(x_{p}\right)\right)=0$. The forgetful homomorphism therefore induces an isomorphism of complexes between

$$
\bigoplus_{x_{1} \in X^{(1)}} G W_{2}^{2}\left(k\left(x_{1}\right), \omega_{x_{1}}\right) \rightarrow \bigoplus_{x_{2} \in X^{(2)}} G W_{1}^{1}\left(k\left(x_{2}\right), \omega_{x_{2}}\right) \rightarrow \bigoplus_{x_{3} \in X^{(3)}} G W\left(k\left(x_{1}\right), \omega_{x_{1}}\right)
$$

and

$$
\bigoplus_{x_{1} \in X^{(1)}} K_{2}\left(k\left(x_{1}\right)\right) \rightarrow \bigoplus_{x_{2} \in X^{(2)}} K_{1}\left(k\left(x_{2}\right)\right) \rightarrow \bigoplus_{x_{3} \in X^{(3)}} K_{0}\left(k\left(x_{1}\right)\right)
$$

To conclude, we prove that $E(3)_{\infty}^{3,-1}=0$. It suffices to show that the cokernel of the homomorphism

$$
\bigoplus_{x_{2} \in X^{(2)}} G W_{2}^{1}\left(k\left(x_{2}\right), \omega_{x_{2}}\right) \longrightarrow \bigoplus_{x_{3} \in X^{(3)}} G W_{1}^{0}\left(k\left(x_{3}\right), \omega_{x_{3}}\right)
$$

is trivial. Lemma 2.4 yields a commutative diagram

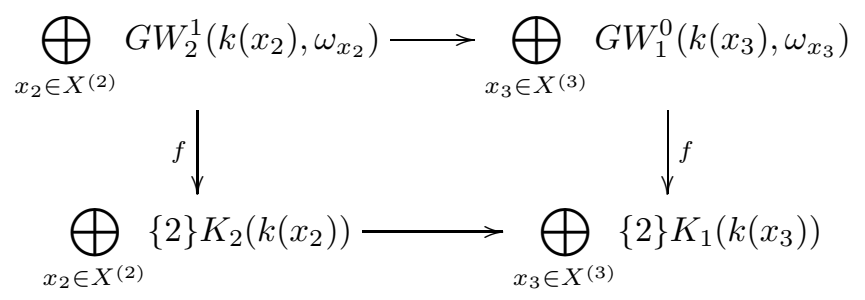

in which the left vertical map is surjective and the right vertical map is an isomorphism. Hence both sequences have the same cokernel.

For any field $F$ and any integer $n \in \mathbb{N}$, define a homomorphism $g_{n}: K_{n}(F) / 2 \rightarrow$ $\{2\} K_{n+1}(F)$ by $\alpha \mapsto\{-1\} \cdot \alpha$. It is clear that $g_{0}$ is an isomorphism, and $g_{1}$ is surjective by [32.

Using the definition of the residue homomorphisms, it is straightforward to check that the diagram

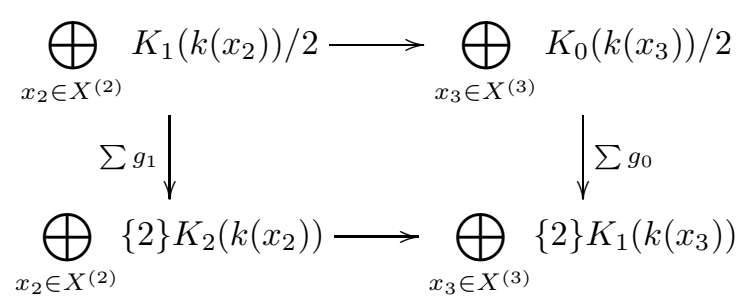

commutes and therefore the cokernels of the rows are isomorphic. The cokernel of the top homomorphism is $C H^{3}(X) / 2$ which is trivial by [11, Lemma 1.2]. The result follows.

\section{Divisibility of $W_{E}$}

In this section, we prove that $W_{E}(R)$ is divisible for a smooth algebra $R$ of dimension 3 over an algebraically closed field. Set $X=\operatorname{Spec}(R)$. In view of Proposition [5.1] it suffices to prove that $H^{2}\left(X, K_{3}\right)$ is divisible. The idea is to use Bloch-Kato (for $K_{2}$ and $K_{1}$ ) and the Bloch-Ogus spectral sequence. 
Proposition 6.1. Let $X$ be a smooth affine threefold over an algebraically closed field $k$. Then $H^{2}\left(X, K_{3}\right)$ is divisible prime to $\operatorname{char}(k)$.

Proof. Let $l$ be a prime number different from $\operatorname{char}(k)$. Consider the exact sequences of sheaves (see [9, proof of Corollary 1.11] for instance)

$$
0 \longrightarrow\left\{l^{n}\right\} K_{3} \longrightarrow K_{3} \longrightarrow l^{n} K_{3} \longrightarrow 0,
$$

and

$$
0 \longrightarrow l^{n} K_{3} \longrightarrow K_{3} \longrightarrow K_{3} / l^{n} K_{3} \longrightarrow 0
$$

Observe that the Gersten resolutions of $\left\{l^{n}\right\} K_{3}$ and $K_{3} / l^{n} K_{3}$ are flasque resolutions of the corresponding sheaves (see [15] for instance), and thus we can use these to compute the corresponding cohomology groups.

Since $K_{0}(F)=\mathbb{Z}$ for any field $F$, we get $H^{3}\left(X,\left\{l^{n}\right\} K_{3}\right)=0$. This yields the following diagram where the line and the column are exact:

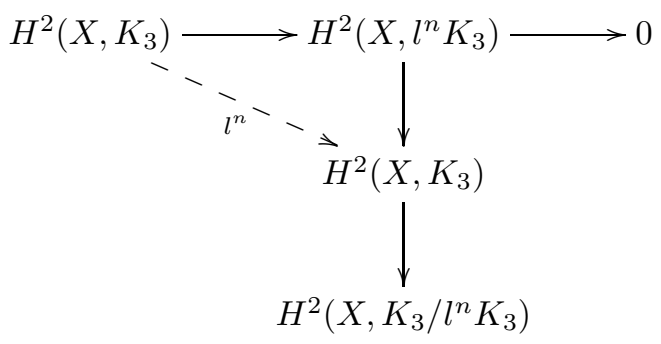

It suffices therefore to prove that $H^{2}\left(X, K_{3} / l^{n} K_{3}\right)=0$ to conclude the divisibility by $l^{n}$.

For any $q, m \in \mathbb{N}$, let $\mathcal{H}^{q}(m)$ be the sheaf associated to the presheaf

$$
U \mapsto H_{e t}^{q}\left(U, \mu_{l^{n}}^{\otimes m}\right)
$$

The Bloch-Ogus spectral sequence ([10]) converges to the étale cohomology groups $H_{e t}^{*}\left(X, \mu_{l^{n}}^{\otimes m}\right)$ and its groups at page 2 are the groups $H_{Z a r}^{p}\left(X, \mathcal{H}^{q}(m)\right)$. These are computed via the Gersten complex

$$
H^{q}\left(k(X), \mu_{l^{n}}^{\otimes m}\right) \stackrel{d_{0}}{\longrightarrow} \bigoplus_{x_{1} \in X^{(1)}} H^{q-1}\left(k\left(x_{1}\right), \mu_{l^{n}}^{\otimes m-1}\right) \longrightarrow \ldots
$$

Using again that $c d\left(k\left(x_{p}\right)\right) \leq 3-p$ for any $x_{p} \in X^{(p)}$ ([31, §4.2, Proposition $11])$, we get $H_{Z a r}^{p}\left(X, \mathcal{H}^{q}(m)\right)=0$ for any $q \geq 4$. Since $X$ is affine, this implies $H^{2}\left(X, \mathcal{H}^{3}(m)\right)=H_{e t}^{5}\left(X, \mu_{l^{n}}^{\otimes m}\right)=0$ by [21, Chapter VI, Theorem 7.2].

Now there is a commutative diagram ([9, Theorem 2.3])

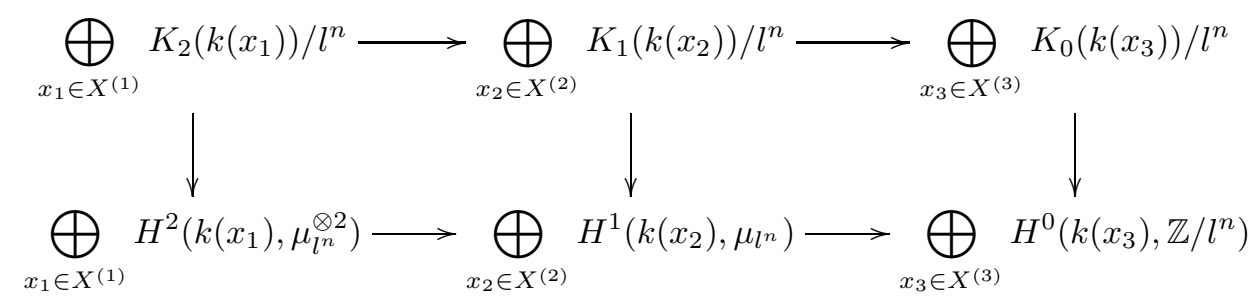

whose vertical maps are isomorphisms $([20])$. This gives

$$
H^{2}\left(X, K_{3} / l^{n}\right)=H^{2}\left(X, \mathcal{H}^{3}(3)\right)=0 .
$$


The result follows.

\section{THE MAIN THEOREM}

In this section, we prove that any stably free module $P$ of rank $(d-1)$ over a $d$-dimensional normal affine algebra $R$ over an algebraically closed field $k$, for which $\operatorname{gcd}((d-1) !, \operatorname{char}(k))=1$, is indeed free.

Recall first that a unimodular row of length $n \geq 2$ over $R$ is a row $a=\left(a_{1}, \ldots, a_{n}\right)$ with $a_{i} \in R$ such that there exist $b_{1}, \ldots, b_{n} \in R$ with $\sum a_{i} b_{i}=1$. We denote by $U m_{n}(R)$ the set of unimodular rows of length $n$ and we consider it as a pointed set with base point $e_{1}:=(1,0, \ldots, 0)$. If $M \in G L_{n}(R)$ and $a \in U m_{n}(R)$, then $a M$ is also unimodular, and in this way $G L_{n}(R)$ acts on $U m_{n}(R)$. If $a$ is any unimodular row, the exact sequence

$$
0 \longrightarrow P(a) \longrightarrow R^{n} \stackrel{a}{\longrightarrow} R \longrightarrow 0
$$

yields a projective module $P(a)$ which is free if and only if there exists $M \in G L_{n}(R)$ such that $a M=e_{1}$. It is clear that any projective module $P$ such that $P \oplus R \simeq R^{n}$ comes from a unimodular row, and it suffices therefore to understand the (pointed) set $U m_{n}(R) / G L_{n}(R)$ to understand stably free modules of rank $n-1$ satisfying $P \oplus R \simeq R^{n}$.

Of course, any subgroup of $G L_{n}(R)$ acts on $U m_{n}(R)$, and one is classically interested in the (pointed) orbit set $U m_{n}(R) / E_{n}(R)$ which classifies unimodular rows up to elementary homotopies. An orbit in $U m_{n}(R) / E_{n}(R)$ is usually called an elementary orbit.

We now recall a lemma of L. N. Vaserstein ([39, Corollary 2]).

Lemma 7.1. Let $\left(a_{1}, a_{2}, a_{3}\right) \in U m_{3}(R), u \in R$, and $R a_{1}+R u=R$. Then the rows $\left(a_{1}, a_{2}, a_{3}\right)$ and $\left(a_{1}, u a_{2}, u a_{3}\right)$ are in the same elementary orbit.

Consequently, by Whitehead's lemma the rows $\left(a_{1}, u a_{2}, u a_{3}\right)$ and $\left(a_{1}, a_{2}, u^{2} a_{3}\right)$ can be seen to be in the same elementary orbit. In particular, if -1 is a square in $R$, then $\left(-a_{1}, a_{2}, a_{3}\right)$ and $\left(a_{1}, a_{2}, a_{3}\right)$ are in the same elementary orbit.

Given $v=\left(v_{0}, v_{1}, v_{2}\right), w=\left(w_{0}, w_{1}, w_{2}\right) \in U m_{3}(R)$, with $v \cdot w^{t}=1$, we shall denote by $V(v, w)$ the class in $W_{E}(R)$ of the $4 \times 4$ skew-symmetric matrix

$$
\left(\begin{array}{cccc}
0 & v_{0} & v_{1} & v_{2} \\
-v_{0} & 0 & w_{2} & -w_{1} \\
-v_{1} & -w_{2} & 0 & w_{0} \\
-v_{2} & w_{1} & -w_{0} & 0
\end{array}\right)
$$

of Pfaffian 1. For simplicity, we may just write $V(v)$, as the class of $V(v, w)$ does not depend on the choice of $w$ such that $v \cdot w^{t}=1$. Associating $V(v)$ to $v$ yields a well defined map $V: U m_{3}(R) / E_{3}(R) \rightarrow W_{E}(R)$ called the Vaserstein symbol (40, $\S 5])$.

Theorem 7.2. Let $R$ be a smooth affine algebra over a field $k$ of cohomological dimension $\leq 1$ which is perfect if the characteristic is 2 or 3 . Then the Vaserstein symbol

is a bijection.

$$
V: U m_{3}(R) / E_{3}(R) \rightarrow W_{E}(R)
$$

Proof. See [27, Corollary 3.5]. 
This bijection gives rise to an abelian group structure on the set $U m_{3}(R) / E_{3}(R)$, when $R$ is as above. We recall Vaserstein's rule in $W_{E}(R)$ (see [40, Theorem 5.2 (iii)]). If $(a, b, c)$ and $(a, x, y)$ are in $U m_{3}(R) / E_{3}(R)$, then

$$
V(a, b, c) \perp V(a, x, y)=V\left(a,(b, c)\left(\begin{array}{cc}
x & y \\
-y^{\prime} & x
\end{array}\right)\right)
$$

for any $x^{\prime}, y^{\prime}$ such that $x x^{\prime}+y y^{\prime}=1$ modulo $(a)$.

Lemma 7.3. Let $R$ be a ring. The following formulas hold in $W_{E}(R)$ :

(i) $V(a, b, c)^{-1}=V\left(-a^{\prime}, b, c\right)$, where $a^{\prime}$ is an inverse of a modulo $(b, c)$.

(ii) $V\left(a b^{2}, c, d\right)=V(a, c, d) \perp V\left(b^{2}, c, d\right)$.

Proof. Since Vaserstein's rule holds in $W_{E}(R)$, it suffices to follow the proof of [38, Lemma 3.5(iii), Lemma 3.5(v)].

We now recall the Antipodal Lemma of Rao in [25, Lemma 1.3.1].

Lemma 7.4. Let $R$ be a ring and $(a, b, c) \in U m_{3}(R)$. Suppose that $(a, b, c)$ and $(-a, b, c)$ are in the same elementary orbit. Then for any $n \in \mathbb{N}$, we have

$$
n V(a, b, c)=V\left(a^{n}, b, c\right)
$$

in $W_{E}(R)$.

Proof. The proof of [25, Lemma 1.3.1] applies mutatis mutandis using the formulas of Lemma 7.3

We now state and prove our main theorem:

Theorem 7.5. Let $R$ be a d-dimensional normal affine algebra over an algebraically closed field $k$ such that $\operatorname{gcd}((d-1) !, \operatorname{char}(k))=1$. If $d=3$, suppose moreover that $R$ is smooth. Then every stably free $R$-module $P$ of rank $d-1$ is free.

Proof. Let $P$ be a stably free module of rank $d-1$. Since the result is clear when $d \leq 2$, we assume that $d \geq 3$. Using Suslin's cancellation theorem [34, Theorem 1], we can suppose that there is an isomorphism $P \oplus R \simeq R^{d}$, and therefore that $P$ is given by a unimodular row $\left(a_{1}, \ldots, a_{d}\right)$. In view of [34, Theorem 2], to prove that $P$ is free it suffices to show that there exists a unimodular row $\left(b_{1}, \ldots, b_{d}\right)$ such that $\left(a_{1}, \ldots, a_{d}\right)=\left(b_{1}^{(d-1) !}, \ldots, b_{d}\right)$ in $U m_{d}(R) / E_{d}(R)$.

Suppose that $d \geq 4$. Let $J$ be the ideal of the singular locus of $R$. Since $R$ is normal, $J$ has height at least 2 and $\operatorname{dim}(R / J) \leq d-2$. It follows from 6 , Theorem 3.5] that $U m_{d}(R / J)=e_{1} E_{d}(R / J)$ and we can therefore assume, performing elementary operations if necessary, that $a_{d} \equiv 1(\bmod J)$ and $a_{1}, \ldots, a_{d-1} \in J$. Using now Swan's Bertini theorem [37, Theorem 1.5], we can perform elementary operations on $\left(a_{1}, \ldots, a_{d}\right)$ such that $B:=R /\left(a_{4}, \ldots, a_{d}\right)$ is either empty, either a non-singular threefold outside the singular locus of $R$. In the first case, the row $\left(a_{4}, \ldots, a_{d}\right)$ is unimodular, and therefore the row $\left(a_{1}, \ldots, a_{d}\right)$ is completable in an elementary matrix. Thus we can restrict to the second case. In this situation, we see that $B$ is actually smooth since $a_{d} \equiv 1(\bmod J)$.

Given a unimodular row $(\bar{a}, \bar{b}, \bar{c})$ on $B$, we can choose lifts $a, b, c \in R$ and consider the unimodular row $\left(a, b, c, a_{4}, \ldots, a_{d}\right)$ on $R$. It is straightforward to check that this gives a well-defined map

$$
U m_{3}(B) / E_{3}(B) \rightarrow U m_{d}(R) / E_{d}(R)
$$


showing that $\left(a_{1}, \ldots, a_{d}\right)$ comes from the unimodular row $\left(\bar{a}_{1}, \bar{a}_{2}, \bar{a}_{3}\right)$ on $B$. We are thus reduced to the case where $R$ is the affine algebra of a smooth threefold. By Theorem 7.2, the set $U m_{3}(R) / E_{3}(R)$ is in bijection with $W_{E}(R)$ and is thus endowed with the structure of an abelian group. Since -1 is a square in $k$, Lemma 7.4 shows that $n \cdot\left(a_{1}, a_{2}, a_{3}\right)=\left(a_{1}^{n}, a_{2}, a_{3}\right)$ in $U m_{3}(R) / E_{3}(R)$ for any $n \in \mathbb{N}$. Now Propositions 5.1 and 6.1 show that $\operatorname{Um}_{3}(R) / E_{3}(R)$ is a divisible group prime to the characteristic of $k$. Since $\operatorname{gcd}((d-1) !, \operatorname{char}(k))=1$, there exists a unimodular row $\left(b_{1}, b_{2}, b_{3}\right) \in U m_{3}(R)$ such that

$$
\left(a_{1}, a_{2}, a_{3}\right)=(d-1) ! \cdot\left(b_{1}, b_{2}, b_{3}\right)=\left(b_{1}^{(d-1) !}, b_{2}, b_{3}\right)
$$

in $U m_{3}(R) / E_{3}(R)$. The result follows.

Remark 7.6. The proof of the theorem actually gives a bit more. Let $R$ be an affine algebra of dimension $d \geq 4$ over an algebraically closed field $k$ such that $\operatorname{gcd}((d-1) !, \operatorname{char}(k))=1$. Let $J$ be the ideal of the singular locus of $R$, and let $\left(a_{1}, \ldots, a_{d}\right)$ be a unimodular row of rank $d$ which is in the elementary orbit of $e_{1}$ in $U m_{d}(R / J) / E_{d}(R / J)$. Then the projective module associated to $\left(a_{1}, \ldots, a_{d}\right)$ is free. To see this, observe that we can use [28, Theorem 2] to reduce to the case of a reduced ring and then apply the proof of the theorem.

To conclude, we derive from the above theorem some stability results for the group $K_{1}(R)$ where $R$ is a smooth affine algebra over an algebraically closed field.

Corollary 7.7. Let $R$ be a smooth algebra of dimension $d \geq 3$ over an algebraically closed field $k$. Assume that $d ! k=k$. Then $S L_{d}(R) \cap E_{d+1}(R)=E_{d}(R)$.

Proof. Let $\sigma \in S L_{d}(R) \cap E_{d+1}(R)$. In view of T. Vorst's theorem in 42, it suffices to show that there exists a matrix $N(X) \in S L_{d}(R[X])$ with the property that $N(0)=I d$ and $N(1)=\sigma$.

Since $1 \perp \sigma \in E_{d+1}(R)$, there exists $\alpha(X) \in G L_{d+1}(R[X])$ with $\alpha(0)=I d$ and $\alpha(1)=1 \perp \sigma$. It follows that the unimodular row $v(X):=e_{1} \alpha(X) \in U m_{d+1}(R[X])$ is congruent to $e_{1}$ modulo $f$, where $f:=X^{2}-X$. We now prove that there exists a matrix $M(X) \in G L_{d+1}(R[X])$ congruent to $I d$ modulo $f$ satisfying the equality $e_{1} M(X)=e_{1} \alpha(X)$. This will prove the corollary since these properties imply that

$$
\alpha(X) M(X)^{-1}=\left(\begin{array}{cc}
1 & 0 \\
\star & N(X)
\end{array}\right)
$$

with $N(X) \in G L_{d}(R[X])$ such that $N(0)=I d$ and $N(1)=\sigma$.

To find $M(X)$, we argue as in [27, Proposition 3.3]. Set $B=R[X, T] /\left(T^{2}-T f\right)$ and write $e_{1} \alpha(X)=e_{1}+\left(X^{2}-X\right) w(X)$, with $w(X) \in R[X]^{d+1}$. Observe that $u(T):=e_{1}+T w(X)$ is a unimodular row of length $d+1$ over $B$ with $u(f)=v(X)$ and $u(0)=e_{1}$. Since $R$ is smooth, it follows that the ideal of the singular locus of $B$ is precisely $(T, f)$. Now $u(T)$ is congruent to $e_{1}$ modulo $T$ and we can use Remark 7.6 to find $\varepsilon(T) \in E_{d+1}(B)$ such that $u(T) \varepsilon(T)$ is of the form $\left(b_{1}^{d !}, \ldots, b_{d+1}\right)$. By [27, Proposition 3.3] or [26, Proposition 5.4], there is a $\delta(T) \in S L_{d+1}(B)$ congruent to $I d$ modulo $f$ such that $u(T) \varepsilon(T)=e_{1} \delta(T)$. Finally, we set $M(X):=\beta(0) \beta(f)^{-1}$ where $\beta(T)=\varepsilon(T) \delta(T)^{-1}$. It is straightforward to check that $M(X)$ satisfies the conditions stated above, and therefore the result is proved.

Corollary 7.8. Let $R$ be a smooth algebra over the algebraic closure $k$ of a finite field of dimension $d \geq 3$. Assume that $d ! k=k$. Then the natural map $S L_{d}(R) / E_{d}(R) \rightarrow S K_{1}(R)$ is an isomorphism. 
Proof. Using [40, Corollary 17.3], we see that $U m_{n}(R)=e_{1} E_{n}(R)$ for $n \geq d$. It follows that $S L_{d}(R) / E_{d}(R) \rightarrow S K_{1}(R)$ is onto. We conclude using Corollary 7.7.

Corollary 7.9. Let $R$ be a smooth algebra of dimension $d \leq 4$ over an algebraically closed field $k$. Assume that $d ! k=k$. Then the Vaserstein symbol

$$
V: U m_{3}(R) / E_{3}(R) \rightarrow W_{E}(R)
$$

is an isomorphism.

Proof. L.N. Vaserstein in [40, Theorem 5.2(c)] has shown that $V$ is onto. Apply [40. Lemma 5.1] to conclude that $V$ is injective as $S L_{4}(R) \cap E(R)=E_{4}(R)$ in view of Corollary 7.7 .

\section{REFERENCES}

[1] Jón Kristinn Arason and Albrecht Pfister. Beweis des Krullschen Durchschnittsatzes für den Wittring. Invent. Math., 12:173-176, 1971.

[2] Paul Balmer. An introduction to triangular Witt groups and a survey of applications. In Algebraic and arithmetic theory of quadratic forms, volume 344 of Contemp. Math., pages 31-58. Amer. Math. Soc., Providence, RI, 2004.

[3] Paul Balmer and Charles Walter. A Gersten-Witt spectral sequence for regular schemes. Ann. Sci. École Norm. Sup. (4), 35(1):127-152, 2002.

[4] Jean Barge and Jean Lannes. Suites de Sturm, indice de Maslov et périodicité de Bott, volume 267 of Progress in Mathematics. Birkhäuser Verlag, Basel, 2008.

[5] H. Bass. K-theory and stable algebra. Inst. Hautes Études Sci. Publ. Math., 22:5-60, 1964.

[6] H. Bass. Algebraic K-theory. W. A. Benjamin, Inc., New York-Amsterdam, 1968.

[7] Hyman Bass. Libération des modules projectifs sur certains anneaux de polynômes. In Séminaire Bourbaki, 26e année (1973/1974), Exp. No. 448, pages 228-354. Lecture Notes in Math., Vol. 431. Springer, Berlin, 1975.

[8] S.M. Bhatwadekar. A cancellation theorem for projective modules over affine algebras over $\mathrm{C}_{1}$-fields. J. Pure Appl. Algebra, 1-3:17-26, 2003.

[9] S. Bloch. Torsion algebraic cycles, $K_{2}$, and Brauer groups of function fields. In The Brauer group (Sem., Les Plans-sur-Bex, 1980), volume 844 of Lecture Notes in Math., pages 75-102. Springer, Berlin, 1981.

[10] Spencer Bloch and Arthur Ogus. Gersten's conjecture and the homology of schemes. Ann. Sci. École Norm. Sup. (4), 7:181-201 (1975), 1974

[11] J.-L. Colliot-Thélène and C. Scheiderer. Zero-cycles and cohomology on real algebraic varieties. Topology, 35(2):533-559, 1996.

[12] J. Fasel and V. Srinivas. A vanishing theorem for oriented intersection multiplicities. Math. Res. Lett., 15(3):447-458, 2008.

[13] J. Fasel and V. Srinivas. Chow-Witt groups and Grothendieck-Witt groups of regular schemes. Adv. Math., 221(1):302-329, 2009.

[14] Jean Fasel. Stably free modules over smooth affine threefolds. Duke Math. J., 156(1):33-49, 2011.

[15] Daniel R. Grayson. Universal exactness in algebraic K-theory. J. Pure Appl. Algebra, 36:139$141,1985$.

[16] Jens Hornbostel. Constructions and dévissage in Hermitian $K$-theory. K-Theory, 26(2):139$170,2002$.

[17] Max Karoubi. Périodicité de la K-théorie hermitienne. In Algebraic K-theory, III: Hermitian $K$-theory and geometric applications (Proc. Conf., Battelle Memorial Inst., Seattle, Wash., 1972), pages 301-411. Lecture Notes in Math., Vol. 343. Springer, Berlin, 1973.

[18] Max Karoubi. Le théorème fondamental de la K-théorie hermitienne. Ann. of Math. (2), 112(2):259-282, 1980.

[19] N. Mohan Kumar. Stably free modules. Amer. J. Math., 107(6):1439-1444, 1985.

[20] A. S. Merkur'ev and A. A. Suslin. K-cohomology of Severi-Brauer varieties and the norm residue homomorphism. Izv. Akad. Nauk SSSR Ser. Mat., 46(5):1011-1046, 1135-1136, 1982. 
[21] James S. Milne. Étale cohomology, volume 33 of Princeton Mathematical Series. Princeton University Press, Princeton, N.J., 1980.

[22] John Milnor. Algebraic K-theory and quadratic forms. Invent. Math., 9:318-344, 1969/1970.

[23] John Milnor. Introduction to algebraic K-theory. Princeton University Press, Princeton, N.J., 1971. Annals of Mathematics Studies, No. 72.

[24] D. Orlov, A. Vishik, and V. Voevodsky. An exact sequence for $K_{*}^{M} / 2$ with applications to quadratic forms. Ann. of Math. (2), 165(1):1-13, 2007.

[25] R.A. Rao. The Bass-Quillen conjecture in dimension three but characteristic $\neq 2,3$ via a question of A. Suslin. Invent. Math., 93(3):609-618, 1988.

[26] R.A. Rao. A stably elementary homotopy. Proc. Amer. Math. Soc., 137:3637-3645, 2009.

[27] Ravi A. Rao and Wilberd van der Kallen. Improved stability for $S K_{1}$ and $W M S_{d}$ of a non-singular affine algebra. Astérisque, 226:11, 411-420, 1994. K-theory (Strasbourg, 1992).

[28] M. Roitman. On unimodular rows. Proc. Amer. Math. Soc., 95:184-188, 1985.

[29] Marco Schlichting. Hermitian K-theory of exact categories. J. K-Theory, 5(1):105-165, 2010.

[30] Marco Schlichting. The Mayer-Vietoris principle for Grothendieck-Witt groups of schemes. Invent. Math., 179(2):349-433, 2010.

[31] Jean-Pierre Serre. Cohomologie galoisienne, volume 5 of Lecture Notes in Mathematics. Springer-Verlag, Berlin, fifth edition, 1994.

[32] A. A. Suslin. Torsion in $K_{2}$ of fields. K-Theory, 1(1):5-29, 1987.

[33] A.A. Suslin. A cancellation theorem for projective modules over algebras. (russian). Dokl. Akad. Nauk S.S.S.R., 236(4):808-811, 1977.

[34] A.A. Suslin. On stably free modules. Math. U.S.S.R. Sbornik, pages 479-491, 1977.

[35] A.A. Suslin. Cancellation for affine varieties. (russian) modules and algebraic groups. Zap. Nauchn. Sem. Leningrad. Otdel. Mat. Inst. Steklov. (L.O.M.I.), 114:187-195, 1982.

[36] A.A. Suslin. Torsion in $K_{2}$ of fields. $K$-Theory, 1(1):5-29, 1987.

[37] R.G. Swan. A cancellation theorem for projective modules in the metastable range. Invent. Math., 27:23-43, 1974.

[38] Wilberd van der Kallen. A module structure on certain orbit sets of unimodular rows. J. Pure Appl. Algebra, 57(3):281-316, 1989.

[39] L.N. Vaserstein. Operations on orbits of unimodular vectors. J. Algebra, 100(2):456-461, 1986.

[40] L.N. Vaserstein and A.A. Suslin. Serre's problem on projective modules over polynomial rings, and algebraic k-theory. Izv. Akad. Nauk S.S.S.R. Ser. Mat., 40(5):993-1054, 1976.

[41] Vladimir Voevodsky. Motivic cohomology with Z/2-coefficients. Publ. Math. Inst. Hautes Études Sci., 98:59-104, 2003.

[42] Ton Vorst. The general linear group of polynomial rings over regular rings. Comm. Algebra, 9:499-509, 1981.

Jean Fasel,

Mathematisches Institut der Universität München,

Theresienstrasse 39,

D-80333 München, Germany

Jean Fasel <jean.fasel@gmail.com>

Ravi A. Rao,

Tata Institute of Fundamental Research,

1, Dr. Homi Bhabha Road, Navy Nagar,

Mumbai 400 005, India

Ravi A. Rao <ravi@math.tifr.res.in>

Richard G. Swan,

University of Chicago,

Chicago, Illinois, 60637, U.S.A.

Richard G. Swan <swan@math.uchicago.edu> 